\title{
Smart parking management using the internet of things
}

Gestión inteligente del estacionamiento empleando internet de las cosas

Author:

Hadi Ghahremani ${ }^{1}$

Shiva Taghipour Eivazi ${ }^{2 * *}$

Amir Aghaei Anvigh ${ }^{3}$

\section{SCIENTIFIC RESEARCH}

How to cite this paper:

Ghahremani. H., Eivazi. S. T., Anvigh. A. A. Smart parking management using the internet of things. Tabriz, Iran. Innovaciencia. 2019; 7 (2): 1-9. DOI:

http://dx.doi.org/10.15649/2346075X.771

Reception date:

Received: 25 January 2019

Accepted: 2 May 2019

Published: 25 October 2019

Keywords:

distance between parking lots, distance between vehicle and a parking lot, number of free parking spaces in the destination parking lot, number of unsuccessful parks, number of parking lot visits, Internet of Things, intelligent parking lot, waiting time

\section{ABSTRACT}

The main requirement of smart cities is intelligent and effective parking system, which currently most parking systems exist based on various technologies such as Internet of Things, so intelligent parking management is gradually becoming a necessity. Hence in this article a new manner is introduced in the form of prioritization, which alters the type of prioritization than the previous methods, so that the different parameters such as the distance between parking lots, distance between vehicle and a parking lot, number of free parking spaces in the destination parking lot, number of unsuccessful parks, and the number of parking lot visits are considered, and in general a system is recommended that helps drivers to find a parking lot with the minimum cost based on the aforesaid parameters. The simulation of proposed approach performed in Arena application and the results of the simulation and its comparison with the previous works suggest that the proposed method results in the better decrease of the waiting time than the existing waiting time in the previous works and contributes to minimize drivers waiting time.

* Corresponding author: Shiva Taghipour Eivazi

1 Department Of Computer Engineering, Tabriz Branch, Islamic Azad University, Tabriz, Iran, Email: hadi.ghahremani73@gmail.com

2 Department Of Computer Engineering, Tabriz Branch, Islamic Azad University, Tabriz , Iran, Email: taghipour@iaut.ac.ir

3 Department Of Information Technology, Tabriz Branch, Islamic Azad University, Tabriz, Iran, Email: amir.aghaei20@gmail.com 


\section{INTRODUCTION}

According to the performed researches the world's biggest parking lot is being constructed by Ferrari Automotive Company with the capacity of forty thousand vehicles in Dubai. The second place after this giant parking lot between the world's biggest parking lots belongs to West Edmonton Promenade in Canada with the capacity of twenty thousand parking spaces. The third place captured by Walt Disney World with the capacity of ten thousands vehicles in America. In keeping with these parking lots capacity, the management of them is of particular importance. The most comprehensive project for management is called parking. Nowadays due to the increase of parking lots capacity and the creation of multilevel parking lots, the parking lot management discussion has been taken into consideration ${ }^{[11}$.

Another point is that nowadays the mechanized and multilevel parking lots are constructed in which without the interference of drivers a parking space is provided for every vehicle as well as approaching to a parked vehicle is mechanized. But using such parking lots is more expensive and on the other hand is not possible in all commercial and recreational zones ${ }^{[2]}$.

In parking lot systems two technologies of parking management and guidance systems are generally used. In parking management system most often the events management topics inside of the parking lot has been taken into consideration. For instance, detection of parking space, parking space reservation, parking lot traffic assessment, evaluation of parking capacity, getting information about how drivers drive inside of a parking lot, getting information about the condition and surroundings in a parking lot, and the circumstance of people inside of a parking lot are the topics associated with the parking management system. In the parking guidance system the topics of vehicles moving direction and their guidance towards parking spaces, exit out of parking lot, or vehicles guidance to other stories are being noticed. In the modern parking management systems both parking guidance and management system topics used since both of these topics together complete the parking management system ${ }^{[3,4]}$.

In the second section of this article the previous works related to the parking management using the internet of things is expressed, and in following the suggested method is introduced. Next the applied example of the suggested method is assessed and the evaluation of the proposed method effectiveness is illustrated by the comparative and evaluation charts and finally the conclusion has been mentioned as the last part of the article.

\section{RELATED WORKS}

The authors ${ }^{[5]}$ recommended in a method for the intelligent management system, using the internet of things based on the cloud, which contributes the users to automatically find free parking lot with the minimum cost and according to distance and the number of total free spaces measures. After user request one parking space is suggested, based on the abovementioned measures, and if it is filled, a new space will be proposed. Algorithm contributes to the improvement of a successful parking probability and minimization of the user waiting time. The new architecture of the intelligent parking system is based on the internet of things, which firstly the algorithm of a system for the search of a vehicle park is considered with the minimum charge, and secondly in case the present parking space is full another mechanism is opted to lead the vehicle to another parking lot. The system considers every parking space as the internet of things and transmits the following data into the data center. The data center acts as a cloud server for the calculation of parking request charges, and these charges are regularly updated and are achievable by vehicles every time. In the car park network each parking lot is a node in the network and each node obtains information from the neighboring node, so it is possible to ensure that the movement of a vehicle will be accomplished with low cost and higher possibility of finding a free space. The recommended system results in the decrease in the number of failed vehicles in the finding of parking space, disorder prevention in the vehicle park system, 
minimization of the wasted time in the finding of a parking space, and decline of the cost in the car transferring into the parking lot.

The authors in ${ }^{[6]}$, presented a parking system by using the image processing technique. The intelligent parking system records the number of every vehicle's license plate for unique identification of every vehicle, this data is then used to calculate the user's payment. In order to show the free or filled spaces of a parking lot some photographs are taken from the parking lot. The main problem of the proposed method is that climate condition affects the camera performance, hence the camera has to be positioned in a spot where to have a perfect view of the parking lot, and on the other hand the proposed method of this article does not guide drivers to park their car.

In ${ }^{[7]}$, the vision-based parking method for the parking lot around which some cameras have been installed, have been introduced, which transmits the real time information to a central database and leads drivers to discover the existing parking spaces or monitor the parked spaces via the wireless communication device. The system displays color images by using the statistical method in which to find the appropriate color every parking space is used, and besides aids to drivers by the colors in order to have proper choice.

Hanif et al ${ }^{[8]}$ represented an intelligent parking system in which users do reserve a parking space by SMS services. In this method one wireless and remote communication device is applied to confirm the details of the reservations such as the number and password. The password for entry into the parking lot was used with the credit of some time periods. A microcontroller called the interface controller has been used for the optimization of the system for the control, and accessibility or ability to save the information of the parking free spaces.
The reservation-based parking system ${ }^{[9]}$ is based on targeting a parking information in real time through an application. This service is for the reservation of a parking space. This enables drivers to retrieve a parking lot information in a certain area of it. Unlike a traditional parking, an intelligent parking extremely increase the reservation service. Parking reservation system can choose the best parking location at the right time. In the data gathering and saving system, parking space condition, reservation time, address, and driver identity are saved in database (for easy access). All data is gathered by the central system and updated, which the changes are reflected in the user's application, so the users can dynamically make a reservation on this basis.

\section{PROPOSE METHOD}

Many methods have been presented in the parking management so far. The most important objection of the previous methods is the ignorance of priority for the driver who has not succeed in his car park as well as the ignorance of the priority for the driver who uses the parking regularly. In this experiment a new method is proposed that changes the type of prioritization than the previous ones, that is how this method of prioritization includes the parameters of distances between parking lots, distance between a vehicle and a parking lot, the number of free parking spaces in the destination parking lot, number of failed parks, and number of the parking lot visits, as well as which parameter should be selected and what coefficient should be applied to, is highly important that the present experiment assesses the various scenarios of these parameters and tries to provide strategies for the optimization of waiting time. In this section some formulas will be applied, so the signs in the formulas have been gathered in following table: 
Table 1: Description of the symbols in the formulas

\begin{tabular}{ccll}
\hline Row & Symbol & \\
\hline $\mathbf{1}$ & $\boldsymbol{d}_{\boldsymbol{i j}}$ & distance between parking lots $P_{j}$ and $P_{i}$ \\
$\mathbf{2}$ & $\boldsymbol{d}_{\boldsymbol{k} j}$ & The distance between the parking lot $P_{j}$ And car $P_{k}$ \\
$\mathbf{3}$ & $\boldsymbol{D}_{\boldsymbol{u} \boldsymbol{p}}$ & $\begin{array}{l}\text { The upper boundary is the distance between parking lots and a global parameter. (The maximum } \\
\text { distance between two parking spaces in the network is placed in this variable }\end{array}$ \\
$\mathbf{4}$ & $\boldsymbol{t}_{\boldsymbol{j}}$ & Number of spaces occupied in the parking lot. \\
$\mathbf{5}$ & $\boldsymbol{T}_{\boldsymbol{u} \boldsymbol{p}}$ & Upper boundary is the parking capacity and a global parameter. (The maximum parking capacity \\
$\mathbf{6}$ & $\boldsymbol{\alpha}$ & Coefficient depends on the length of the path between the two parking lots. \\
$\mathbf{7}$ & $\boldsymbol{\gamma}$ & Coefficient depends on the length of the path between the car and the parking lot \\
$\mathbf{8}$ & $\boldsymbol{\beta}$ & Coefficient depends on the number of free parking spaces in the destination parking lot. \\
$\mathbf{9}$ & $\boldsymbol{\omega}$ & Coefficient depends on the number of parking spaces used. \\
$\mathbf{1 0}$ & $\boldsymbol{\theta}$ & Coefficient depends on the number of unsuccessful parks. \\
$\mathbf{1 1}$ & $\boldsymbol{\omega}_{\boldsymbol{c}}$ & Number of parking use \\
$\mathbf{1 2}$ & $\boldsymbol{\boldsymbol { \theta } _ { \boldsymbol { l } }}$ & Number of unsuccessful parks \\
\hline
\end{tabular}

Once a driver decides to park his car, a parking lot with the minimum charge is suggested by using formula 1.

$$
F_{k j}(\gamma, \beta, \omega, \theta)=\gamma * d_{k j}+\beta * \frac{t_{j}}{T_{u p}}+\omega * \frac{1}{\omega_{c}}+\theta * \frac{1}{\theta_{l}}
$$

The above formula $F_{k j}(\gamma, \beta, \omega, \theta)$ depends on the following parameters:

1- The distance between the vehicle and the parking lot, 2- number of occupied parking spaces in the destination parking lot, 3 - number of times that the parking lot is used, 4- number of failed parks.

In case the capacity of the recommended parking lot according to formula 1 is completed before arriving the driver and parking the vehicle, a new parking lot is recommended by using formula 2 that is based on the minimum charge.

$F_{i j}(\alpha, \beta, \omega, \theta)=\alpha * \frac{d_{i j}}{D_{u p}}+\beta * \frac{t_{j}}{T_{u p}}+\omega * \frac{1}{\omega_{c}}+\theta * \frac{1}{\theta_{l}}$

The above formula $F_{k j}(Y, \beta, \omega, \theta)$ depends on the following parameters:

1- The distance between two parking lot, 2- number of occupied parking spaces in the destination parking lot, 3- number of times that the parking lot is used, 4- number of failed parks

The previous approaches only comprise the parameters of distance between parking lots, the distance between the vehicle and the parking lot, and the number of free parking space in the destination parking lot, so in order to improve the failures of the previous works, the type of prioritization in compare to the previous works has been changed in 
the proposed method and includes the parameters of distances between parking lots, distance between a vehicle and a parking lot, the number of free parking spaces in the destination parking lot, number of failed parks, and number of the parking lot visits. In other words in the proposed approach two parameters of the loyal customers and the people who have used the parking lot for many times, and the people who could not have been able to park their cars in the designated places by the system, are taken into consideration as well. The analysis of information is conducted according to the abovementioned formulas and the existing parameters. The issue that which parameters ought to be used and what coefficient should be considered is so important, but on the other hand the total coefficient of the parameters should be 1 and the range of every coefficient of the formula parameters is between 0 and 1 .

\section{EXAMINING AN EXAMPLE}

A city has three parking lots and the driver of vehicle $A$ requests a parking space to park his car. The optimal case in terms of waiting time is offered according to the proposed method, as well as the proposed method and previous works are compared and evaluated.

Each parking number is as Pi symbol and the specification of three parking lots are as follows:

Table 2: Description of N Symbols

\begin{tabular}{cccc}
\hline Row & Specifications & Symbol & Total Parking Capacity \\
\hline $\mathbf{1}$ & Number of parking lot number 1 & N1 & 100 \\
$\mathbf{2}$ & Number of parking lot number 2 & N2 & 70 \\
$\mathbf{3}$ & Number of parking lot number 3 & N3 & 120 \\
\hline
\end{tabular}

Table 3: Descriptions of NI symbols

\begin{tabular}{cccc}
\hline Row & Specifications & Symbol & Number of occupied parking spaces \\
\hline $\mathbf{1}$ & Numberof occupied parking spaces number 1 & NI1 & 40 \\
$\mathbf{2}$ & Number of occupied parking spaces number 2 & NI2 & 20 \\
$\mathbf{3}$ & Number of occupied parking spaces number 3 & NI3 & 70 \\
\hline
\end{tabular}

Table 4: Description of DI symbols

\begin{tabular}{cccc}
\hline Row & \multicolumn{1}{c}{ Specifications } & Symbol & Distance $(\mathbf{k m})$ \\
\hline $\mathbf{1}$ & The distance between the parking lot 1 and 2 & D12 & 50 \\
$\mathbf{2}$ & The distance between the parking lot 1 and 3 & D13 & 20 \\
$\mathbf{3}$ & The distance between the parking lot 2 and 3 & D23 & 40 \\
\hline
\end{tabular}

It is supposed that each parking lot is a node in the network. Each node has one side table for the information protection about the current condition of the network and another side table for each node containing the related information to the neighboring nodes such as the current number of free parking space and so forth, which are directly are related to it. Each node sends a message to its neighboring node after parking or exiting a vehicle from the parking space. This message comprises the information about its free spaces. The neighboring node which receives this message updates the related tables of its neighbor. 
Table 5: Parking Table Number 1

\begin{tabular}{ccccc}
\hline Row & Neighbor & Space & Parking capacity & Occupied space \\
\hline $\mathbf{1}$ & Parking number 2 & 50 & 70 & 20 \\
$\mathbf{2}$ & Parking number 3 & 20 & 120 & 70 \\
\hline
\end{tabular}

Table 6: Parking side number 2

\begin{tabular}{ccccc}
\hline Row & Neighbor & Space & Parking capacity & Occupied space \\
\hline $\mathbf{1}$ & Parking number 1 & 50 & 100 & 40 \\
$\mathbf{2}$ & Parking number 3 & 40 & 120 & 70 \\
\hline
\end{tabular}

Table 7: Parking table number 3

\begin{tabular}{ccccc}
\hline Row & Neighbor & Space & Parking capacity & Occupied space \\
\hline $\mathbf{1}$ & Parking number 1 & 20 & 100 & 40 \\
$\mathbf{2}$ & Parking number 2 & 40 & 70 & 20 \\
\hline
\end{tabular}

The position of vehicle A and parking is as follows:

Table 8: Vehicle conditions A according to the parameters $(\gamma)$

\begin{tabular}{cccc}
\hline Row & Specifications & Symbol & Space \\
\hline $\mathbf{1}$ & The distance between vehicle A and parking number 1 & $D_{A 1}$ & 30 \\
$\mathbf{2}$ & The distance between vehicle A and parking number 2 & $D_{A 2}$ & 15 \\
$\mathbf{3}$ & The distance between vehicle A and parking number 3 & $D_{A 3}$ & 12 \\
\hline
\end{tabular}

Table 9: Vehicle conditions A by parameter $(\beta)$

\begin{tabular}{cccc}
\hline Row & Specifications & Symbol & Parking capacity \\
\hline $\mathbf{1}$ & Number of parking lots number 1 & N1 & 100 \\
$\mathbf{2}$ & Number of parking lots number 2 & N2 & 70 \\
$\mathbf{3}$ & Number of parking lots number 3 & N3 & 120 \\
\hline
\end{tabular}

Table 10: Vehicle conditions A by parameter $(\omega)$

\begin{tabular}{cllc}
\hline Row & \multicolumn{1}{c}{ Specifications } & Symbol & number \\
\hline $\mathbf{1}$ & Number of car use A from parking number 1 & $\omega_{A 1}$ & 30 \\
$\mathbf{2}$ & Number of car use A from parking number 2 & $\omega_{A 2}$ & 15 \\
$\mathbf{3}$ & Number of car use A from parking number 3 & $\omega_{A 3}$ & 1 \\
\hline
\end{tabular}


Table 11: Vehicle conditions A by parameter $(\theta)$

\begin{tabular}{clcc}
\hline Row & \multicolumn{1}{c}{ Specifications } & Symbol & number \\
\hline $\mathbf{1}$ & Number of unsuccessful park car A in parking lot number 1 & $\theta_{A 1}$ & 10 \\
$\mathbf{2}$ & Number of unsuccessful park car A in parking lot number 2 & $\theta_{A 2}$ & 8 \\
$\mathbf{3}$ & Number of unsuccessful park car A in parking lot number 3 & $\theta_{A 3}$ & 1 \\
\hline
\end{tabular}

Vehicle A requests a parking space in its location (according to abovementioned circumstance).

1) Based on formula 1 , the parameters coefficients are considered the values of $F_{k j}(0.25,0.25,0.25,0.25)$ :

$$
\begin{aligned}
& F_{P 1 j}(0.25,0.25,0.25,0.25)=0.25 * 30+0.25 * \frac{40}{120}+0.25 * \frac{1}{30}+0.25 * \frac{1}{10}=7.61 \\
& F_{P 2 j}(0.25,0.25,0.25,0.25)=0.25 * 15+0.25 * \frac{20}{120}+0.25 * \frac{1}{15}+0.25 * \frac{1}{8}=3.83 \\
& F_{P 3 j}(0.25,0.25,0.25,0.25)=0.25 * 12+0.25 * \frac{70}{120}+0.25 * \frac{1}{1}+0.25 * \frac{1}{1}=3.64
\end{aligned}
$$

The minimum waiting time is for $F_{P 2 j}$ status, so the vehicle $\mathrm{A}$ is directed to parking lot number 3 .

2) Based on formula 2, the parameters coefficients are considered the values of $F_{k j}(0.5,0.5)$ :

$$
\begin{aligned}
& F_{P 1 j}(0.5,0.5)=0.5 * 30+0.5 * \frac{40}{120}=15.166 \\
& F_{P 2 j}(0.5,0.5)=0.5 * 15+0.5 * \frac{20}{120}=7.583 \\
& F_{P 3 j}(0.5,0.5)=0.5 * 12+0.5 * \frac{70}{120}=6.291
\end{aligned}
$$

Minimum waiting time for mode $F_{P 3 j}$, So the car A is driven into parking number 3.

In the next section the proposed method is simulated in the environment.

\section{SIMULATION AND RESULTS}

Arena application is a discrete event simulation and automation software, which is one of the best computer simulation software, and it is an application software with high modeling capability and a perfect tool for simulation studies, and supports all steps of one simulation study, and it has been manufactured by Rockwell Automation Company in 2000 and since December 2016, in version 15, has had remarkable advance on the optimization, animation, and modeling of processes with giant information (Using 64-bit operation).

This application is used for the projects of intelligent parking lot, manufacturing, packaging, food and beverage products, supply chain, call center, customers' services and so forth.

Arena is a good application that simulates many real-time systems such as parking systems. The main 
blocks of Arena models structure are modules. Flow diagram modules describe the dynamic processes in a model. The different types of flowchart modules are; creation, discard, processing, decision making, classification, decollate, allocation and record. Costs determine resources, and lines. They can also regulate variables and other types of numerical values and the words associated with the whole model.

In the simulation of the parking system, Arena particularly supports the random distributions from the stages of entry and services such as Poisson, normal, exponential, triangular, uniform, beta, gamma, and logarithmic distributions. This issue permits the statistical and average values of parameters calculations used for the performance evaluation such as a vehicle waiting time for parking space request and the vehicle average time in the parking system. Most of the previous researches have demonstrated that the results of simulation in Arena is close to those who are real in action. Considering the abovementioned advantages, Arena has been chosen as the simulation tool in this experiment.

Two parking lots have been evaluated with the following characteristics and according to the descriptions in the previous section in Arena simulation software.

Table 12: Number of park parking spaces

\begin{tabular}{clcc}
\hline Row & Specifications & Symbol & Parking capacity \\
\hline 1 & Number of parking lots number 1 & N1 & 5 \\
2 & Number of parking lots number 2 & N2 & 5 \\
\hline
\end{tabular}

Table 13: Number of vacant parking spaces

\begin{tabular}{cllc}
\hline Row & \multicolumn{1}{c}{ Specifications } & Symbol & Number of vacant parking spaces \\
\hline $\mathbf{1}$ & Number of vacant parking spaces number 1 & NI1 & 5 \\
$\mathbf{2}$ & Number of vacant parking spaces number 2 & NI2 & 5 \\
\hline
\end{tabular}

Table 14: Simulation setting parameters

\begin{tabular}{ccc}
\hline Parameter name & unit & Value \\
\hline The number of vehicles entered each car park & $70,80,90,100$ & Vehicles \\
Coefficient $V, \alpha, \beta, \omega, \theta$ & Between 0 and 1 & - \\
\hline
\end{tabular}

The simulation results have been illustrated in figure 1 that with regard to this figure the proposed method reduces the waiting time. 


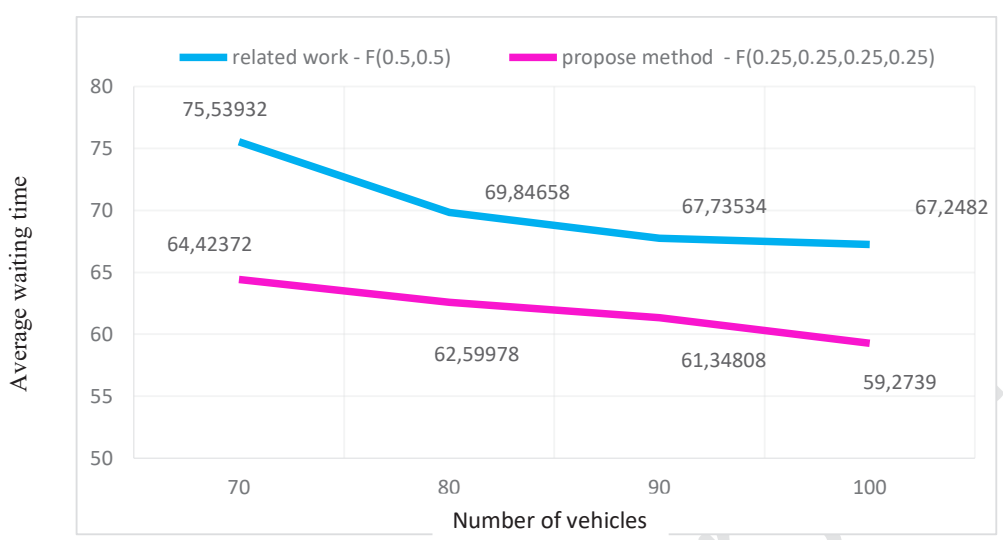

Figure 1: Simulation Results

\section{CONCLUSION}

In this article the intelligent parking management system was assessed using the internet of things with the purpose of reducing waiting time. First a brief introduction mentioned, next the different related researches in the field of the intelligent parking management system collected using the internet of things and then the desired proposed method was mentioned, and eventually the proposed method was described further by giving an example. In the last section the proposed method was assessed in Arena stimulation application and ultimately the assessment of the proposed method effectiveness was explained via the comparative and evaluation charts.

\section{REFERENCES}

1. Hadi Ghahremani, Mehrdad Abdi," Navigation Improvement in Parking Management System with Internet of Things", conference on civil engineering, architecture \& urbanism of the Islamic countries, isc, (2017)

2. Aniket Gupta, Sujata Kulkarni, Vaibhavi Jathar, Ved Sharma, Naman Jain, " Smart Car Parking Management System Using IoT", American Journal of Science, Engineering and Technology, (2017). https://doi.org/10.11648/j.ajset.20170204.13

3. Muhammad Babar, Fahim Arif," Smart Urban Planning using Big Data Analytics to contend with the Interoperability in Internet of Things", Future Generation Computer Systems, ELSEVIER, (2017).

https://doi.org/10.1016/j.future.2017.07.029
4. Sarang Deshpande, "M-Parking: Vehicle Parking Guidance System using Hierarchical Wireless Sensor Networks", Annual Consumer Communications \& Networking Conference (CCNC), IEEE, (2016). https://doi.org/10.1109/CCNC.2016.7444888

5. Thanh Nam Pham, Ming Fong Tsai, Duc Binh Nguyen, Chyi Ren Dow, Der Jiunn Deng, "A Cloud Based Smart Parking System Based on Internet of Things Technologies", International Journal Of IEEE Access, (2016). https://doi.org/10.1109/access.2015.2477299

6. Hilal Al Kharusi, Ibrahim Al Bahadly, "Intelligent Parking Management System Based on Image Processing", World Journal of Engineering and Technology, (2014). https://doi.org/10.4236/wjet.2014.22006

7. Lin S.F, Chen Y.Y, Liu, S.C, "A vision-based parking lot management system", Proceedings of IEEE International Conference System Man Cybernetics, (2007). https://doi.org/10.1109/ICSMC.2006.385314

8. Hanif, N.H.H.M, Badiozaman, M.H., Daud, H., "Smart parking reservation system using short message services (SMS)", International Conference on Intelligent and Advanced Systems (ICIAS), (2010)

9. Wand and W. He, "A reservation based smart parking system", Workshop on Cyber Physical networking systems, (2011). https://doi.org/10.1109/ICIAS.2010.5716179 